\title{
ARTICLE
}

\section{Germline mutations in the PAF1 complex gene CTR9 predispose to Wilms tumour}

Sandra Hanks ${ }^{1, \star}$, Elizabeth R. Perdeaux ${ }^{1, \star}$, Sheila Seal ${ }^{1}$, Elise Ruark ${ }^{1}$, Shazia S. Mahamdallie ${ }^{1}$, Anne Murray ${ }^{1}$, Emma Ramsay ${ }^{1}$ Silvana Del Vecchio Duarte1, Anna Zachariou1, Bianca de Souza ${ }^{1,2}$, Margaret Warren-Perry ${ }^{1}$, Anna Elliott ${ }^{1}$, Alan Davidson ${ }^{3}$, Helen Price ${ }^{4}$, Charles Stiller ${ }^{5}$, Kathy Pritchard-Jones ${ }^{6}$ \& Nazneen Rahman ${ }^{1,2}$

Wilms tumour is a childhood kidney cancer. Here we identify inactivating CTR9 mutations in 3 of 35 Wilms tumour families, through exome and Sanger sequencing. By contrast, no similar mutations are present in 1,000 population controls $(P<0.0001)$. Each mutation segregates with Wilms tumour in the family and a second mutational event is present in available tumours. CTR9 is a key component of the polymerase-associated factor 1 complex which has multiple roles in RNA polymerase II regulation and is implicated in embryonic organogenesis and maintenance of embryonic stem cell pluripotency. These data establish CTR9 as a Wilms tumour predisposition gene and suggest it acts as a tumour suppressor gene.

\footnotetext{
${ }^{1}$ Division of Genetics and Epidemiology, Institute of Cancer Research, London SM2 5NG, UK. ${ }^{2}$ Cancer Genetics Unit, Royal Marsden Hospital NHS Foundation Trust, London SM2 5PT, UK. ${ }^{3}$ Department of Paediatric Haematology-Oncology, Red Cross Children's Hospital, University of Cape Town, Cape Town 7700, South Africa. ${ }^{4}$ Department of Paediatrics, Great Western Hospital, Swindon SN3 6BB, UK. ${ }^{5}$ Childhood Cancer Research Group, University of Oxford, Oxford OX1 2JD, UK. ${ }^{6}$ Molecular Haematology and Cancer Biology Unit, Institute of Child Health, University College London, London WC1E 6BT, UK.

* These authors contributed equally to this work. Correspondence and requests for materials should be addressed to N.R. (email: rahmanlab@icr.ac.uk).
} 
W ilms tumour is the most common paediatric renal cancer, affecting 1 in 10,000 children. It is often described as an embryonal tumour as it arises from embryonal cells in which growth and/or differentiation has become dysregulated during development. Eighty per cent of individuals with Wilms tumour are diagnosed by 5 years of age and diagnosis after 15 years is extremely rare. Treatment of Wilms tumour is very successful with 5 -year overall survival of $\sim 90 \%$ (ref. 1).

About $2 \%$ of Wilms tumour patients have one or more relatives that have also had Wilms tumour ${ }^{1,2}$. In most Wilms tumour families, there are no other clinical features or cancers and the majority are consistent with an autosomal dominant mode of inheritance with incomplete penetrance. A small proportion of familial cases are due to WT1 mutations, 11 p15 epigenetic defects or autosomal recessive conditions that include Wilms tumour, such as mosaic variegated aneuploidy syndrome and certain types of Fanconi anaemia ${ }^{2}$. Two familial Wilms tumour loci have been mapped by genome-wide linkage analysis to chromosomes $17 \mathrm{q} 12-21$ and 19q13, but the causative genes remain elusive ${ }^{3,4}$. Furthermore, families unlinked to either locus have been reported $^{5}$. Thus, to date, the cause(s) of the majority of familial Wilms tumours is unknown.

In this study, we use exome and Sanger sequencing in familial Wilms tumour to identify new genes that predispose to
Wilms tumour. We identify inactivating Cln three requiring 9 (CTR9) mutations in three of 35 Wilms tumour families, establishing CTR9, which encodes a key component of the polymerase-associated factor complex (PAF1c), as a Wilms tumour predisposition gene.

\section{Results}

We first performed exome sequencing of lymphocyte DNA from 12 affected individuals from six unrelated, non-syndromic Wilms tumour families (Table 1, Methods). We generated 39,660,686$106,571,869$ reads per sample, with an average of 59,575,502 reads across the 12 samples. As many cancer predisposition genes are tumour suppressor genes, inactivated by rare protein truncating variants (PTVs), we used a PTV prioritization method to identify candidate truncating mutations for further investigation ${ }^{6}$. Specifically, we used NextGENe software (SoftGenetics) to identify and annotate all variants in the exome data. We excluded any gene with more than one PTV in 48 exomes of individuals with other conditions that were sequenced and analyzed in parallel, through the same pipelines. We next identified PTVs in the remaining genes, that were present in all affected individuals within a family and stratified the genes according to the number of families that harboured diseasesegregating PTVs (Methods).

\section{Table 1 | Wilms tumour families included in study.}

\section{Family ID Relationship of relatives affected with Wilms tumour}

FAM0072 Two siblings

FAM0091 Two siblings

FAM0123 Two siblings

FAM0349 Two half first cousins once removed (parent's half first cousin)

FAM0477 Parent-child and possible history of more distantly affected relatives

FAM0480 Two siblings

FAM0481 Three first cousins

FAM0484 Two first cousins. Mothers are sisters and fathers are brothers

FAM0485 Two first cousins and one first cousin once removed (first cousin of obligate carrier parents)

FAM0486 Parent-child. Parent has affected half-sibling and first cousin

FAM0487 Uncle-child

FAM0488 Two half siblings

FAM0489 Two first cousins

FAM0491 Two first cousins

FAM0492 Parent-child

FAM0493 Two siblings

FAM0498 Parent-children (two children)

FAM0499 Uncle-child (via unaffected obligate carrier father)

FAM0500 Two first cousins

FAM0501 Two individuals are second cousins (one set of grandparents are siblings) and third cousins (one set of grandparents are first cousins)

FAM0504 Parent-child

FAM0507 Two half second cousins (grandparents are half siblings)

FAM0508 Parent-child

FAM0509 Two first cousins once removed (parent's first cousin)

FAM0510 Parent-child

FAM0678 Two siblings

FAM0689 Uncle-child (via unaffected obligate carrier mother)

FAM2006 Parent-children (two children)

FAM2084 Two second cousins

FAM2097 Two second cousins

FAM3679 Two siblings

FAM3727 Two siblings

FAM5673 Parent-child

FAM5737 Two second cousins

FAM5804 Parent-child

\begin{tabular}{|c|c|}
\hline $\begin{array}{l}\text { Number of WT } \\
\text { cases analyzed }\end{array}$ & Method of analysis \\
\hline 1 & CTR9 Sanger sequencing \\
\hline 1 & CTR9 Sanger sequencing \\
\hline 2 & CTR9 Sanger sequencing \\
\hline 2 & Exome sequencing \\
\hline 2 & CTR9 Sanger sequencing \\
\hline 1 & CTR9 Sanger sequencing \\
\hline 2 & CTR9 Sanger sequencing \\
\hline 1 & CTR9 Sanger sequencing \\
\hline 1 & CTR9 Sanger sequencing \\
\hline 1 & CTR9 Sanger sequencing \\
\hline 1 & CTR9 Sanger sequencing \\
\hline 2 & Exome sequencing \\
\hline 2 & CTR9 Sanger sequencing \\
\hline 1 & CTR9 Sanger sequencing \\
\hline 1 & CTR9 Sanger sequencing \\
\hline 2 & Exome sequencing \\
\hline 2 & Exome sequencing \\
\hline 2 & CTR9 Sanger sequencing \\
\hline 1 & CTR9 Sanger sequencing \\
\hline 2 & CTR9 Sanger sequencing \\
\hline 2 & CTR9 Sanger sequencing \\
\hline 2 & Exome sequencing \\
\hline 1 & CTR9 Sanger sequencing \\
\hline 2 & CTR9 Sanger sequencing \\
\hline 2 & CTR9 Sanger sequencing \\
\hline 2 & CTR9 Sanger sequencing \\
\hline 1 & CTR9 Sanger sequencing \\
\hline 2 & CTR9 Sanger sequencing \\
\hline 1 & CTR9 Sanger sequencing \\
\hline 1 & CTR9 Sanger sequencing \\
\hline 2 & CTR9 Sanger sequencing \\
\hline 2 & Exome sequencing \\
\hline 2 & CTR9 Sanger sequencing \\
\hline 1 & CTR9 Sanger sequencing \\
\hline 2 & CTR9 Sanger sequencing \\
\hline
\end{tabular}

WT, Wilms tumour. 
Table 2 | gDNA and cDNA primer sequences and sizes.

\begin{tabular}{|c|c|c|c|c|}
\hline PCR primer & Forward sequence $\left(5^{\prime}-3^{\prime}\right)$ & Reverse sequence $\left(5^{\prime}-3^{\prime}\right)$ & PCR product size (bp) & Multiplex group \\
\hline Exon 1 & GAGCCGTCACTCACCTCTG & AAGGAGGATGCTCTCGCTTC & 235 & A \\
\hline Exon 2 & TGTTGATTATATTCACGAAAAGCAG & GCAGCCAAGCTGGCTATTAC & 276 & $\mathrm{~B}$ \\
\hline Exon 3 & CTAATCCCTGTGCCAGACAAC & TCCTTGAAATTCACCTGGAG & 856 & C \\
\hline Exon 5 & TGTTCTGTGTTTTCAAGTATTTCTCAC & TCTTTTCATAACTTCATAAGCAACTG & 304 & $\mathrm{~B}$ \\
\hline Exon 6 & GTTATACTGAGGTTAATTTTGGGG & AGACACATCTGGCTCCAAGAG & 356 & C \\
\hline Exon 7 & GCTTCTAATCCGTTTTAGTGTCTG & AACAAACTCTATAATTTGGAGGGG & 368 & D \\
\hline Exon 12 & CCTAGGGGAGGCTAAGGTAGG & CTGGAGAAATGGGGACATTAG & 441 & A \\
\hline Exon 13 & СCTTTGGGACTTTTCTGTTCC & CAAAACCAGGAAGATGTAGCC & 318 & B \\
\hline Exon 14-15 & CAGTAATCAGCTATTGTGGGAAG & AAACAACTACATTGATCACATTTTAAG & 546 & C \\
\hline Exon 16 & TTGTTTCAAATGAATACTTTCAGAGG & ATGACAGGGCCAGAATGG & 380 & $A$ \\
\hline Exon $17-18$ & TTGCAATGCCATTTTGCTAC & GCATTTCAGACAAAATCGGG & 591 & B \\
\hline Exon 19 & GCAAACCTTTTCTCAGACTTTG & СCTCTGTTCCCTACTGTGGC & 262 & C \\
\hline cDNA primer & \multicolumn{2}{|l|}{ Primer sequence } & \multicolumn{2}{|c|}{ Expected PCR product size (bp) } \\
\hline Exon $7 \mathrm{~F}$ & \multicolumn{2}{|c|}{ TGGCAAATCACTTTTTTCTTCAA } & \multicolumn{2}{|c|}{499} \\
\hline Exon $10 R$ & \multicolumn{2}{|c|}{ TTTGTGCCAATTCAATCCAA } & \multicolumn{2}{|c|}{387} \\
\hline Exon $8 \mathrm{~F}$ & \multicolumn{2}{|c|}{ СССТCCATGCATTCCATAAT } & \multicolumn{2}{|c|}{387} \\
\hline Exon $11 R$ & \multicolumn{2}{|c|}{ AGGATTCGTGTTGCTGTTCC } & \multicolumn{2}{|r|}{499} \\
\hline
\end{tabular}

The analysis identified only one gene, CTR9, that contained two different disease-segregating PTVs in two of the Wilms tumour families, and no PTVs in the 48 non-Wilms exomes. We identified two different CTR9 PTVs in four individuals from two unrelated families. The mean coverage across the mutations was $70 \times$ and the mutant-read percentage was $50 \%$. We also confirmed the mutations by Sanger sequencing (Table 2, Methods).

Fam0488 includes two half-sisters who developed Wilms tumour at 33 and 39 months (Fig. 1a, Methods). A heterozygous CTR9 nonsense mutation, c.106C $>\mathrm{T}$; p.Q36X, was present in each half-sister (Fig. 1b). It is assumed that their father was also a mutation carrier, but this cannot be confirmed, as he died before a sample could be obtained.

Fam3727 includes sisters who developed Wilms tumour as infants at 9 and 8 months (Fig. 1a, Methods). A heterozygous essential splice-site mutation c.1194 $+2 \mathrm{~T}>\mathrm{C}$ was detected in both sisters and was inherited from their unaffected father (Fig. 1b). The mutation is predicted to abolish the exon 9 splice-site, which we confirmed by minigene analysis and complementary DNA (cDNA) sequencing, showing that it results in the deletion of exon 9 (p.320_398del78) (Table 2, Fig. 2a,c, Methods).

We next Sanger sequenced the 25 exons and intron-exon boundaries of CTR9 in DNA from 43 individuals with familial Wilms tumour from 29 families (Tables 1 and 2, Methods). We identified another exon 9 splice-site mutation, c.1194+3A $>$ C, in Fam0484, which includes two cousins affected by Wilms tumour at 14 and 36 months (Fig. 1a, Methods). The cousins are related through both parents as their fathers are brothers and their mothers are sisters (Fig. 1a). Both fathers carry the CTR9 splice-site mutation. Analysis of cDNA demonstrated that the mutation results in aberrant splicing and deletion of exon 9, causing the same 78 amino acid deletion detected in Fam3727 (Fig. 2b,c). Thus, in total, our analyses identified CTR9 mutations in three of 35 Wilms tumour families.
Tumour material was available from two individuals. The Wilms tumour from Fam3727, proband 2 showed loss of the wild-type CTR9 allele (Fig. 1b). By contrast, the germline mutation was heterozygous in tumour DNA from Fam0484, proband 1. We therefore sequenced CTR9 in the tumour DNA and identified a somatic truncating mutation, c.3487A $>\mathrm{T}$; p.R1163X (Fig. 1b). While the phase of this mutation in relation to the germline splicing mutation could not be determined, the presence of two mutations in the tumour is consistent with CTR9 being a tumour suppressor gene that requires both alleles to be inactivated for oncogenesis to proceed. It should be noted however that the somatic CTR9 mutation is close to the end of the gene and therefore may not have significant functional impact. It is also noteworthy that only one truncating somatic CTR9 mutation has been reported in 4,745 tumours in which the gene has been analyzed in the COSMIC database (http://cancer.sanger.ac.uk/cosmic). Taken together, the available data suggest that the somatic mutation in Fam0484 is not a random passenger event and is likely to be causally related to the development of the Wilms tumour.

To further evaluate the likely pathogenicity of the mutations we had identified, we sequenced CTR9 in 1,000 UK population controls by exome sequencing. No mutation predicted to truncate or alter CTR9 splicing was identified (Table 3, Methods). Furthermore, no CTR9 splicing or truncating mutations have been reported in 8,588 European American or 4,402 African American individuals sequenced through the NHLBI GO Exome Sequencing Project (http://evs.gs.washington.edu/EVS/). These data add further evidence for the association of CTR9 mutations with familial Wilms tumour $(3 / 35$ versus $0 / 1,000 ; P<0.0001)$. Thus our results provide compelling evidence that CTR9 is a Wilms tumour predisposition gene and strongly suggest it functions as a tumour suppressor gene.

Mutations in some cancer predisposition genes contribute appreciably to both familial and non-familial cases, whereas for 
a

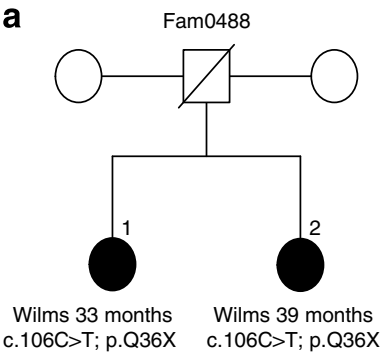
c.106C>T; p.Q36X c.106C>T; p.Q36X
Fam3727
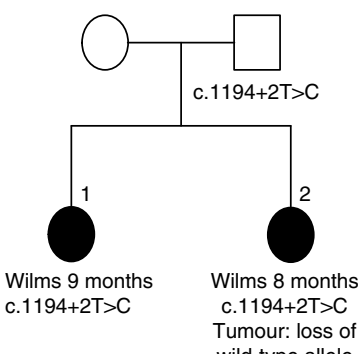

wild-type allele
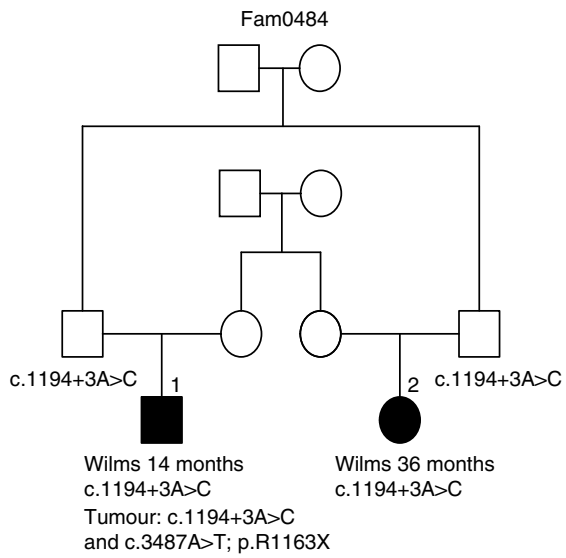

b

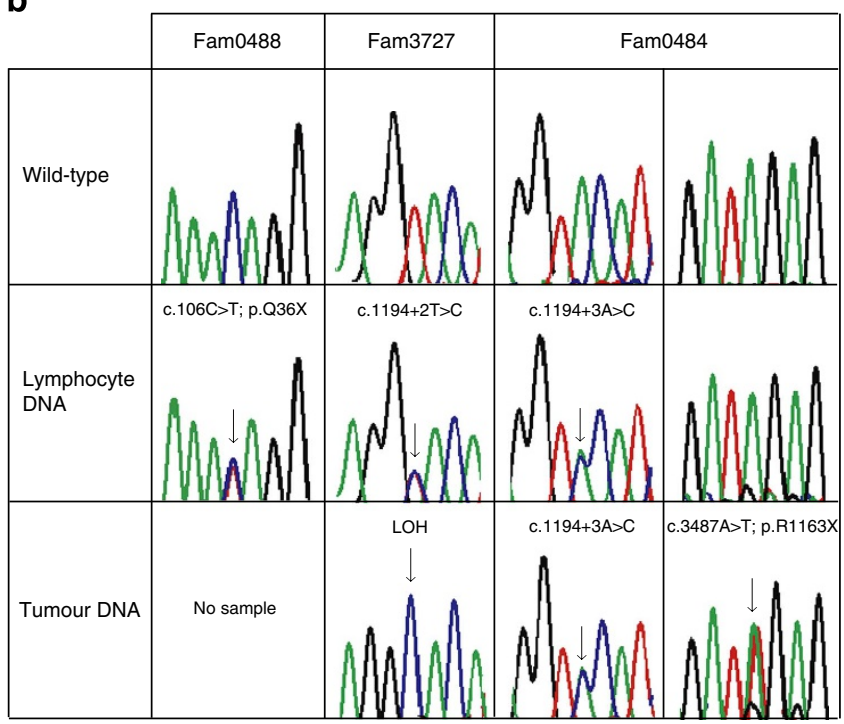

Figure 1 | Germline CTR9 mutations in familial Wilms tumour. (a) Pedigrees of three Wilms tumour families with germline CTR9 mutations. The age at diagnosis and mutation are shown under the relevant individuals. (b) Sequencing chromatograms showing mutations in blood and tumour DNA and corresponding wild-type sequence from a control.

a

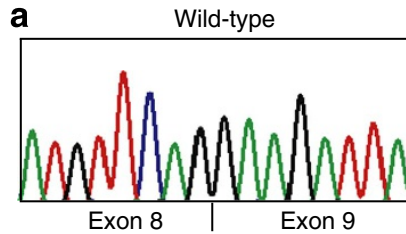

Fam3727: c. $1194+2 T>C$

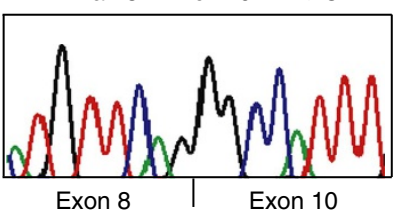

b Wild-type

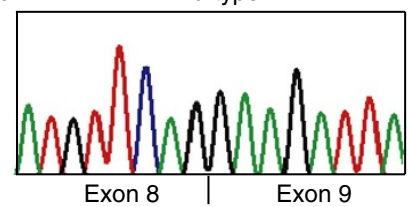

Fam0484: c. $1194+3 A>C$

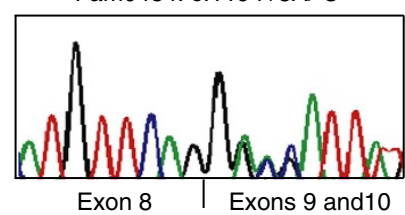

C

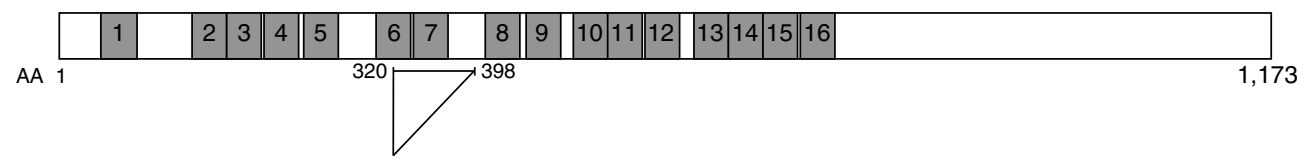

\begin{tabular}{|l|l|l|l|l|l|l|l|l|l|l|l|l|l|l|l|l|l|l|}
\hline & 1 & & 2 & 3 & 4 & 5 & & & 8 & 9 & 10 & 11 & 12 & 13 & 14 & 15 & 16 & \\
\hline AA 1 & \multicolumn{10}{|c|}{} & \\
\hline
\end{tabular}

Figure 2 | Splicing CTR9 mutations cause in-frame deletion of CTR9 TPR domains. (a) Sequencing chromatograms from Reverse Transcription-PCR analysis of RNA from HEK293 cells, transiently transfected with CTR9 minigene splicing constructs containing the c.1194 +2 T $>$ C mutation identified in Fam3727, showing monoallelic deletion of exon 9. (b) cDNA analysis from Fam0484 (proband 2), who is heterozygous for c.1194 + 3A >C, demonstrates that exon 9 is deleted on one allele. (c) Schematic structures of normal and mutant forms of CTR9 protein showing tetratricopeptide repeat domains (shaded boxes). The c.1194 +2T >C and c.1194 + 3A > C splice-site mutations result in an in-frame deletion of amino acids 320-398 containing two tetratricopeptide repeats.

others the contribution to non-familial cases is small. To evaluate the contribution of CTR9 to non-familial Wilms tumour, we sequenced the coding exons and intron-exon boundaries of the gene in 587 individuals with Wilms tumour and no history of relatives with Wilms tumour. No truncating or splicing mutations were identified. Thirty-two intronic, synonymous or non-synonymous variants were detected, but none are predicted to be pathogenic and the spectrum of variation in Wilms tumour 
Table 3 | Non-pathogenic CTR9 variants identified in Wilms tumour cases and controls.

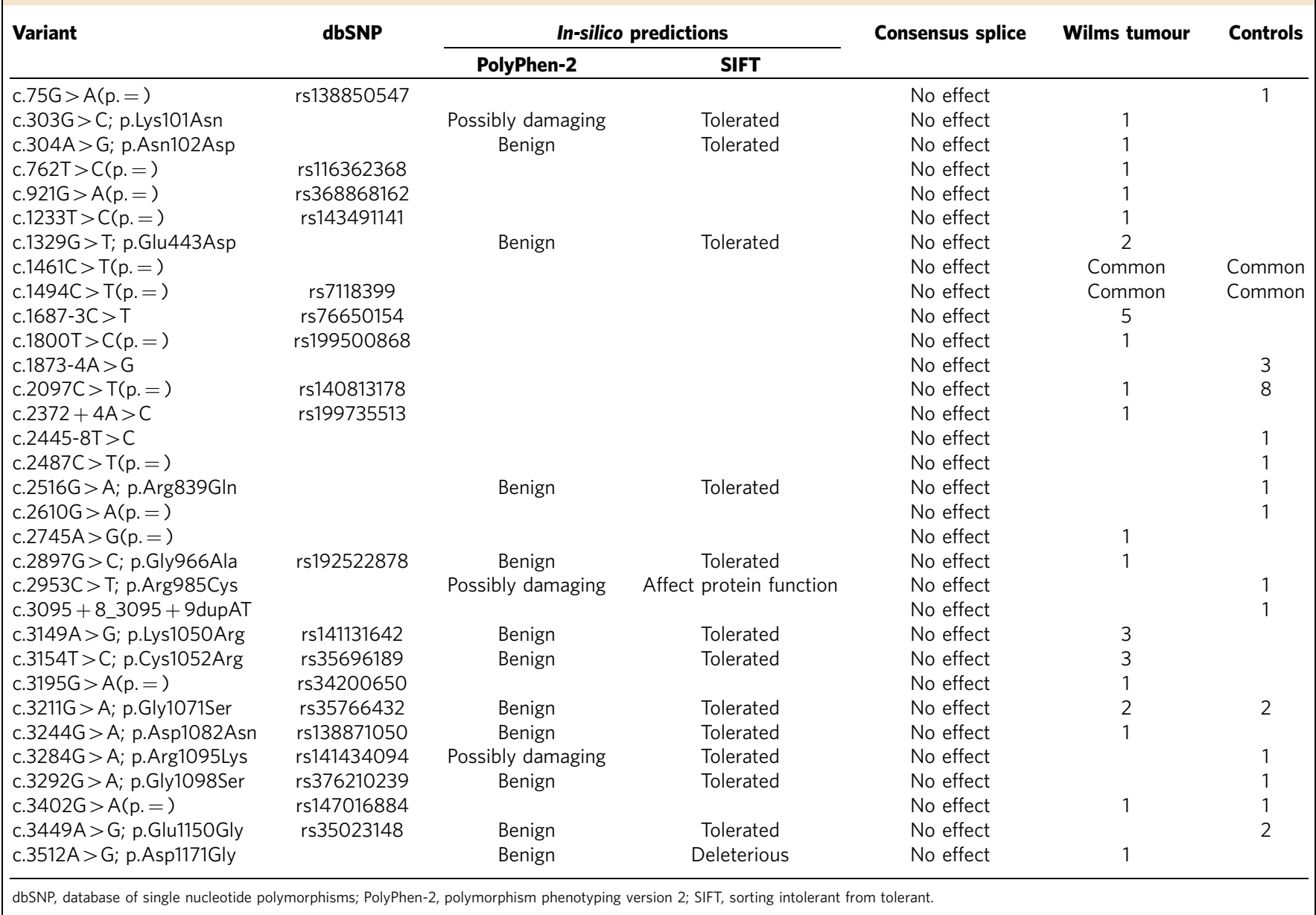

cases was similar to that in the 1,000 population controls. (Table 3). Thus CTR9 mutations appear to be a very rare cause of Wilms tumour, and typically result in familial clustering of the disease.

CTR9 is located at $11 \mathrm{p} 15.3$ and encodes a 1,173 amino acid protein. It is widely expressed, including in foetal and adult kidney, and shows evolutionary conservation throughout eukaryotes $^{7,8}$. CTR9 contains multiple tetratricopeptide repeats (TPR), a versatile protein-protein interaction domain that can act as interaction scaffolds in multi-protein complexes involved in diverse cellular processes 9 . Two of the three mutations we identified are distinct splicing mutations that result in the same in-frame deletion of exon 9, which includes 78 amino acids and encompasses two of the TPR protein-protein interaction domains. It is tempting to speculate that this mutant protein has specific dysfunction that results in cancer predisposition. However, the third germline mutation generates a stop codon that likely results in a truncated product that lacks all the TPR repeats, or nonsense-mediated RNA decay and haploinsufficiency. Functional analyses to explore the impact of the mutations on CTR9 function will be of interest.

CTR9 is a core component of PAF1c, which has multiple roles in RNA Polymerase II regulation ${ }^{10}$ (Fig. 3). PAF1c is a multiprotein complex including PAF1, LEO1, CDC73 (also known as parafibromin), CTR9, RTF1 and WDR61 (also known as SKI8). The complex plays important roles in a wide range of biological processes, including the initiation, elongation and termination of gene transcription and transcription-coupled histone modifications such as $\mathrm{H} 2 \mathrm{~B}$ monoubiquitination and
H3K4 and H3K36 methylation ${ }^{7,8}$. Through these critical regulatory functions, PAF1c influences many essential cellular processes including gene silencing and activation, messenger RNA processing, protein synthesis, DNA repair and cell cycle progression $^{7,8}$. More recently, PAF1c, particularly CTR9 and RTF1, have been shown to have important roles in organ development during embryogenesis ${ }^{11}$ and the maintenance of embryonic stem cell identity ${ }^{12}$.

PAF1c has also been implicated in oncogenesis 8 . Most importantly, $C D C 73$ is a tumour suppressor gene and cancer predisposition gene ${ }^{13,14}$. Heterozygous inactivating CDC73 mutations have been shown to cause hyperparathyroidism-jaw tumour syndrome (OMIM 145001) and to predispose to cancer $^{13,14}$. The associated clinical features are variable and include hyperparathyroidism, parathyroid cancer, ossifying fibromas of the jaw, renal abnormalities and uterine tumours. Parathyroid cancer is the most frequent malignant manifestation, and it is estimated that $20-30 \%$ of sporadic parathyroid cancers are due to germline $C D C 73$ mutations ${ }^{13}$. Intriguingly, Wilms tumour is a rare association of CDC73 mutations, having been reported in three individuals, one of whom presented with biallelic Wilms tumour at the exceptionally late age of 53 years $^{2}$.

The results presented here identify CTR9 as the second PAF1c component that is a cancer predisposition gene. They also further highlight the high heterogeneity of genetic predisposition of Wilms tumour and indicate that additional Wilms tumour predisposition genes must exist. The genes encoding other components of PAF1c: PAF1, LEO1, RTF1 and WDR61, are all 


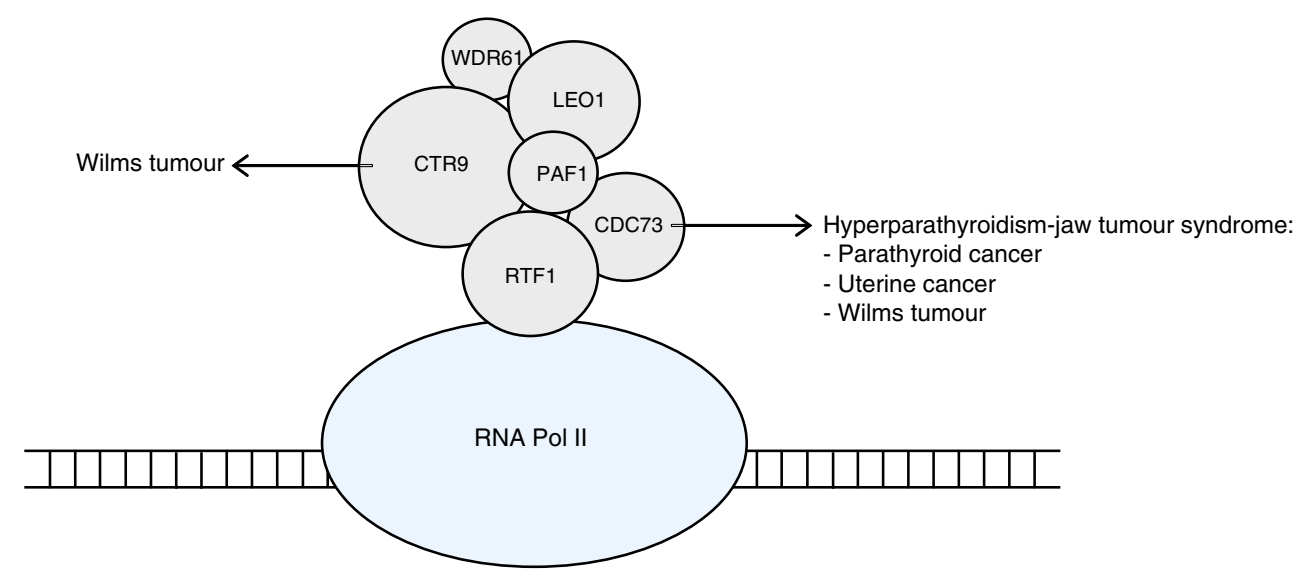

Figure 3 | Schematic scale diagram of PAF1c and RNA Pol II. PAF1c consists of six subunits: PAF1, LEO1, CDC73, CTR9, RFT1 and WDR61. CTR9 and $C D C 73$ are cancer predisposition genes, mutations in which cause Wilms tumour and hyperparathyroidism-jaw tumour syndrome, respectively. RNA Pol II, RNA polymerase II.

highly credible candidate predisposition genes for Wilms tumour and other cancers.

\section{Methods}

Patients and samples. The families were recruited through the Factors Associated with Childhood Tumours (FACT) collaboration as detailed in Supplementary Note 1. The study was approved by the UK National Research Ethics Service-London Multicentre Committee (05/MRE02/17) and informed consent was given by all participants, or their parents as appropriate. We included two DNA samples from each of the six families in the exome sequencing experiment. WT1 mutations and 11 p15 epigenetic analysis had been performed and were negative. The cases were all non-syndromic, with no evidence of syndromic conditions associated with Wilms tumour such as mosaic variegated aneuploidy or Fanconi anaemia. We also included 43 DNA samples from affected individuals of 29 families (all WT1 and 11 p15 negative) and samples from 587 non-familial, unselected Wilms tumour cases, in the CTR9 Sanger sequencing experiment (Table 1). In the CTR9 mutationpositive families we obtained additional samples from relatives and tumour samples, where available. DNA was extracted from whole blood using standard protocols. DNA was extracted from tumour samples using the Phusion Human Specimen Direct PCR Kit (Finnzyme) according to the manufacturer's instructions.

Control samples. We used lymphocyte DNA from 1,000 population-based controls obtained from the 1958 Birth Cohort Collection, a continuing follow-up of persons born in the United Kingdom in 1 week in 1958. Biomedical assessment was undertaken during 2002-2004 at which blood samples and informed consent were obtained for the creation of a genetic resource (http://www.cls.ioe.ac.uk/).

Case reports of CTR9 mutation-positive families. Fam0488 includes two half-sisters with Wilms tumour (Fig. 1a). Proband 1 presented with a right-sided abdominal mass at 33 months and proband 2 presented with a right-sided abdominal mass at 39 months. Both were Wilms tumour, though the histological subtype was not specified. There were no additional clinical features in either child and no family history of childhood cancer. Sequencing of WT1 and epigenetic analyses of $11 \mathrm{p} 15$ by Methylation-Specific Multiplex Ligation-dependent Probe Amplification (MS-MLPA) were normal. The children were successfully treated and subsequently lost to follow-up. Their father is presumed to carry the CTR 9 mutation. He did not have Wilms tumour and died of a dissecting thoracic aortic aneurysm.

Fam3727 includes two sisters, both diagnosed with Wilms tumour as infants (Fig. 1a). Proband 1 was diagnosed with a triphasic, stromal-predominant, Stage I Wilms tumour of the right kidney at 9 months. She was also found to have a large left ureterocele and a small dysplastic left kidney. Subsequently, while pregnant, she was found to have a unilateral duplicated left ureter. Proband 2 was diagnosed with blastemal-predominant, Stage 1 Wilms tumour of the right kidney at 8 months. There were no syndromic features in either child. Karyotypes were normal, and sequencing of WT1 and epigenetic analyses of 11p15 by MS-MLPA were normal. Both sisters are now adults and remain well.

Fam0484 includes first cousins, a boy and a girl, related through both parents; their fathers are brothers and their mothers are sisters (Fig. 1a). Proband 1 was diagnosed with blastemal-predominant, Stage III Wilms tumour of the left kidney at 14 months. Proband 2 was diagnosed with blastemal-predominant, Stage I Wilms tumour of the left kidney at 36 months. Sequencing of WT1 and epigenetic analyses of $11 \mathrm{p} 15$ by MS-MLPA were normal. The cousins are now adults and have remained well.

Exome sequencing. We prepared DNA libraries from $1.5 \mu \mathrm{g}$ blood-derived genomic DNA using the Paired-End DNA Sample Preparation Kit (Ilumina). DNA was fragmented using Covaris technology and the libraries were prepared without gel size selection. We performed target enrichment using the TruSeq Exome Enrichment Kit (Illumina) by targeting $62 \mathrm{mb}$ of the human genome. The captured DNA libraries were PCR amplified using the supplied paired-end PCR primers. Sequencing was performed with an Illumina HiSeq2000 generating $2 \times 101 \mathrm{bp}$ reads.

Exome variant calling. For the Wilms tumour samples, we undertook read mapping and variant analysis using NextGENe software (SoftGenetics) version 2.10 as previously described ${ }^{15,16}$. We generated $39,660,686-106,571,869$ reads per sample, with an average of 59,575,502 reads across the 12 samples. Variants were called using the default NextGENe software mutation calling filters.

For the control samples, we mapped sequencing reads to the human reference genome (hg19) using Stampy version 1.0.14 (ref. 15). Duplicate reads were flagged using Picard version 1.60 (http://picard.sourceforge.net). Median coverage of the target at $15 \times$ was $91 \%$ across the 1,000 individuals, with a median of $47,240,000$ reads mapping to the target. Variant calling was performed with Platypus version 0.1 .5 (ref. 16).

Exome data analysis. For each family, we first removed all variants, which appeared in only one of the affected individuals in each family, such that only disease-segregating variants were further evaluated. We next utilized the PTV prioritization method ${ }^{6}$. This is a gene-based strategy that aims to prioritize potential disease-associated genes for follow-up by leveraging two properties of PTVs: (1) the strong association of rare truncating variants with disease, and (2) collapsibility; different PTVs within a gene typically result in the same functional effect and can be equally combined. Specifically, we identified nonsense mutations, coding insertions or deletions that would generate translational frameshifts and insertions, deletions or base substitutions that would disrupt consensus splice residues. We then excluded any gene with more than one PTV in 48 exomes of individuals with other conditions that were sequenced and analyzed in parallel through the same pipelines. This was on the assumption that familial Wilms tumour is a very rare condition, and thus mutations in a Wilms tumour predisposing gene would not be present in unrelated individuals without Wilms tumour. We then stratified the remaining genes according to the number of families that harboured disease-segregating PTVs. We implemented the analyses in the statistical software package R. Scripts are available on request.

CTR9 Sanger sequencing. We screened CTR9 for mutations using Sanger sequencing. Amplifying primers, flanking exons and intron-exon boundaries, were designed using Exon-Primer from the UCSC genome browser (http://genome. ucsc.edu/). Primer sequences are given in Table 2. PCR reactions were performed in multiplex using the QIAGEN Multiplex PCR Kit (QIAGEN) according to the manufacturer's instructions. Amplicons were unidirectionally sequenced using the BigDyeTerminator Cycle sequencing kit and an ABI 3730 automated sequencer (Life Technologies). We analyzed sequencing traces using Mutation Surveyor software (SoftGenetics) and by visual inspection. We confirmed all mutations by bidirectional sequencing from a fresh aliquot of stock DNA. Samples from members of CTR9 mutation-positive families were tested for the family mutation 
by direct sequencing of the appropriate exon. We also sequenced tumour DNA where available to confirm whether the mutation identified in constitutional DNA was present in the tumour.

In-silico analysis of identified variants. We computed the predicted effects of CTR9 non-synonymous variants on protein function using polymorphism phenotyping version 2 (PolyPhen-2) and sorting intolerant from tolerant (SIFT). All variants (intronic and coding) were also analyzed for their potential effect on splicing. Variants were analyzed using three splice prediction algorithms: NNsplice, MaxEntScan and HumanSplicingFinder. These analyses were performed with Alamut software (Interactive Biosoftware).

cDNA analysis of splice-site mutations. Using genomic DNA from both probands of Fam3727, we amplified the variant and the flanking sequence of interest and inserted the fragment into the multiple cloning sites of vector pcDNA3.1/ myc-His(A) (Life Technologies). The vector contains a cytomegalovirus promoter and an ampicillin cassette for selection in bacteria. We used DH5 $\alpha$ competent cells for transformation and selected clones for the correct inserts. We purified plasmids containing wild-type CTR9 sequence or the c. $1194+2 \mathrm{~T}>\mathrm{C}$ mutation using a QIAprep Spin MiniPrep Kit (QIAGEN) and transfected the products into HEK293 cells using Lipofectamine reagent (Life Technologies) according to the manufacturer's instructions. After $48 \mathrm{~h}$, we harvested the cells and extracted RNA using the RNeasy Mini Kit (QIAGEN). We also extracted RNA from whole blood provided by proband 2 of Fam0484 using the PAXgene Blood RNA Kit (QIAGEN) according to the manufacturer's instructions. In all cases we synthesized cDNA using the ThermoScript RT-PCR System (Life Technologies) with random hexamers and $1 \mu \mathrm{g}$ of total RNA. We amplified the mutation regions using cDNA-specific primers (Table 2) and sequenced the PCR products as described above.

\section{References}

1. Pritchard-Jones, K. et al. Treatment and outcome of Wilms' tumour patients: an analysis of all cases registered in the UKW3 trial. Ann. Oncol. 23, 2457-2463 (2012).

2. Scott, R. H., Stiller, C. A., Walker, L. \& Rahman, N. Syndromes and constitutional chromosomal abnormalities associated with Wilms tumour. J. Med. Genet. 43, 705-715 (2006)

3. Rahman, N. et al. Evidence for a familial Wilms' tumour gene (FWT1) on chromosome 17q12-q21. Nat. Genet. 13, 461-463 (1996).

4. McDonald, J. M. et al. Linkage of familial Wilms' tumor predisposition to chromosome 19 and a two-locus model for the etiology of familial tumors. Cancer Res. 58, 1387-1390 (1998).

5. Rapley, E. A. et al. Evidence for susceptibility genes to familial Wilms tumour in addition to WT1, FWT1 and FWT2. Br. J. Cancer 83, 177-183 (2000).

6. Ruark, E. et al. Mosaic PPM1D mutations are associated with predisposition to breast and ovarian cancer. Nature 493, 406-410 (2013).

7. Jaehning, J. A. The Paf1 complex: platform or player in RNA polymerase II transcription? Biochim. Biophys. Acta 1799, 379-388 (2010).

8. Tomson, B. N. \& Arndt, K. M. The many roles of the conserved eukaryotic Paf1 complex in regulating transcription, histone modifications, and disease states. Biochim. Biophys. Acta 1829, 116-126 (2013).

9. Allan, R. K. \& Ratajczak, T. Versatile TPR domains accommodate different modes of target protein recognition and function. Cell Stress Chaperones 16, 353-367 (2011).

10. Mueller, C. L. \& Jaehning, J. A. Ctr9, Rtf1, and Leo1 are components of the Paf1/RNA polymerase II complex. Mol. Cell. Biol. 22, 1971-1980 (2002).

11. Akanuma, T., Koshida, S., Kawamura, A., Kishimoto, Y. \& Takada, S. Paf1 complex homologues are required for Notch-regulated transcription during somite segmentation. EMBO Rep. 8, 858-863 (2007).
12. Ding, L. et al. A genome-scale RNAi screen for Oct4 modulators defines a role of the Paf1 complex for embryonic stem cell identity. Cell Stem Cell 4, 403-415 (2009).

13. Carpten, J. D. et al. HRPT2, encoding parafibromin, is mutated in hyperparathyroidism-jaw tumor syndrome. Nat. Genet. 32, 676-680 (2002).

14. Rich, T. A. GeneReviews (University of Washington, 2008; updated 2012).

15. Lunter, G. \& Goodson, M. Stampy: a statistical algorithm for sensitive and fast mapping of Illumina sequence reads. Genome Res. 21, 936-939 (2011).

16. Rimmer, A., Phan, H., Mathieson, I., Zamin, I., Twigg, S. R. F., WGS500 Consortium, Wilkie, A. O. M. \& McVean, G. Integrating mapping-, assemblyand haplotype-based approaches for calling variants in clinical sequencing applications. Nat. Genet. (2012).

\section{Acknowledgements}

We thank the families for their participation in our research and the physicians and nurses that recruited them. Samples were collected through the Factors Associated with Childhood Tumours (FACT) study, which is a Children's Cancer and Leukaemia Group (CCLG) Study (the UK National Research Ethics Service Reference: 05/MRE02/17). We are grateful to Darshna Dudakia, Jessie Bull, Rosy Williams, Usha Kini and Judith Kingston for assistance in recruitment. The CCLG receives funding from the UK Department of Health, the National Cancer Intelligence Network, the Scottish Government and Children with Cancer UK. We acknowledge use of services provided by the Institute of Cancer Research Genetics Core Facility, which is managed by Sandra Hanks and Nazneen Rahman. We acknowledge NHS funding to the Royal Marsden/ICR NIHR Biomedical Research Centre. This research was supported by the Wellcome Trust (088804/Z/09/Z), Cancer Research UK (C8620_A9024) and the Institute of Cancer Research, UK.

\section{Author contributions}

S.H., E.R.P., S.S., S.S.M., A.M., E.Ra. and S.D.V.D. performed the molecular analyses. E.Ru. and A.E. performed bioinformatics analyses. A.D., H.P., C.S. and K.P.-J. provided samples and data, which was coordinated by A.Z., B.S. and M.W.-P. N.R. designed and oversaw all aspects of the study and wrote the paper with contributions from the other authors

\section{Additional information}

Accession codes: The patient exome sequence data has been deposited in the European Genome-phenome Archive (EGA) under the accession code EGAS00001000904. Access to this data is through the ICR Genetic Susceptibility Team data access committee.

Supplementary Information accompanies this paper at http://www.nature.com/ naturecommunications

Competing financial interests: The authors declare no competing financial interests

Reprints and permission information is available online at http://npg.nature.com/ reprintsandpermissions/

How to cite this article: Hanks, S. et al. Germline mutations in the PAF1 complex gene CTR9 predispose to Wilms tumour. Nat. Commun. 5:4398 doi: 10.1038/ncomms5398 (2014).

cc) $(\$$ This work is licensed under a Creative Commons AttributionNonCommercial-NoDerivs 4.0 International License. The images or other third party material in this article are included in the article's Creative Commons license, unless indicated otherwise in the credit line; if the material is not included under the Creative Commons license, users will need to obtain permission from the license holder to reproduce the material. To view a copy of this license, visit http:// creativecommons.org/licenses/by-nc-nd/4.0/ 